\title{
APPROXIMATE SOLUTIONS FOR A CLASS OF INTEGRAL EQUATIONS*
}

\author{
BY \\ RICHARD LATTER \\ The RAND Corporation
}

1. Introduction. Exact solutions for integral equations of the type

$$
f(x)=g(x)+\lambda \int_{0}^{a} d y k(x-y) f(y), \quad 0 \leq x \leq a,
$$

are, in general, exceedingly difficult to obtain, except for particularly simple forms for $k(x-y)$ such as a sum of polynomials times exponentials. In the present discussion, it will be shown that by an extension of the Wiener-Hopf technique, it is possible to obtain approximate solutions as well as approximate eigenvalues for this equation. The accuracy of the solutions will be shown to improve exponentially with increasing order of approximation. Application of the method is made to the cases wherein $k(x)=$ $e^{-|x|}$, for which case an exact solution is obtained, and $k(x)=1 /(2 \pi)^{1 / 2} \exp \left(-1 / 2 x^{2}\right)$, for which case the lowest eigenvalue is obtained approximately.

2. Fourier transform of (1). The first step in the present method consists in obtaining the generalized Fourier transform (Titchmarsh [1]) of (1). For this purpose, it is assumed that $f(x)=0\left(e^{c|x|}\right)(c<1), g(x)=0\left(e^{d|x|}\right)(d<1), k(x)=0\left(e^{-|x|}\right)$, and $k(x)=k(-x)$, $k(x)$ real; and that these functions belong to $L_{2}(-\infty, \infty)$. The following arguments treat (1) as if it holds for all $x$. This constitutes no limitation since if (1) is assumed to apply only for $0 \leq x \leq a$, then the conditions $k(x)=0$ for $|x|>a$ and $g(x)=0$ for $x<-a$ and for $x>2 a$ may be arbitrarily required. From these conditions it is observed that $f(x)=0$ for $x<-a$ and for $x>2 a$ and therefore $f(x), g(x)$ and $k(x)$ automatically satisfy the assumed growth conditions. To simplify the subsequent formulas, the following quantities are defined and their region of analyticity indicated:

$$
\begin{array}{ll}
\text { (i) } & f_{+}(w)=\int_{a}^{\infty} d x e^{i x w} f(x) \quad(\operatorname{Im} w>c), \\
\text { (ii) } \quad f_{a}(w)=\int_{0}^{a} d x e^{i x w} f(x) \quad(\operatorname{Im} w>-\infty), \\
\text { (iii) } \quad f_{-}(w)=\int_{-\infty}^{0} d x e^{i x w} f(x) \quad(\operatorname{Im} w<-c), \\
\text { (iv) } \quad K(w)=\int_{-\infty}^{\infty} d x e^{i x w} k(x) \quad(-1<\operatorname{Im} w<1), \\
\text { (v) } \quad g_{+}(w)=\int_{a}^{\infty} d x e^{i x w} g(x) \quad(\operatorname{Im} w>d), \\
\text { (vi) } \quad g_{a}(w)=\int_{0}^{a} d x e^{i x w} g(x) \quad(\operatorname{Im} w>-\infty), \\
\text { (vii) } \quad g_{-}(w)=\int_{-\infty}^{0} d x e^{i x w} g(x) \quad(\operatorname{Im} w<-d) .
\end{array}
$$

*Received October 1, 1956; revised manuscript received January 21, 1957. 
In terms of these quantities and by the Fourier inversion formula, (1) becomes

$$
\begin{aligned}
\int_{i \mu-\infty}^{i \mu+\infty} d w e^{-i x w}\left[f_{-}(w)-g_{-}(w)\right]+ & \int_{i r-\infty}^{i \tau+\infty} d w e^{-i x w}\left[f_{+}(w)\right. \\
& \left.-g_{+}(w)-g_{a}(w)-(\lambda K(w)-1) f_{a}(w)\right]=0,
\end{aligned}
$$

where $\tau>-1 ; c, d<\tau<1$, and $\mu<-c,-d$. By a theorem due to Titchmarsh ([1], p. 255), (2) yields directly for the transform of (1)

$$
[\lambda K(w)-1] f_{a}(w)=f_{+}(w)+f_{-}(w)-g_{+}(w)-g_{-}(w)-g_{a}(w),
$$

where in the region $|\operatorname{Im} w|<1$, the functions $f_{-}(w)-g_{-}(w)$ and $f_{+}(w)-g_{+}(w)-$ $g_{a}(w)-(\lambda K(w)-1) f_{a}(w)$ are regular and tend to zero uniformly as $|\mathrm{Rl} w| \rightarrow \infty$ in any interior strip. Since $f_{a}(w), g_{a}(w)$ and $K(w)$ are also regular and tend to zero uniformly as $|\operatorname{Rl} w| \rightarrow \infty$ in any strip in $|\operatorname{Im} w|<1$, the same properties hold for $f_{+}(w)-$ $g_{+}(w)$.

3. Integral equations for $f_{+}(w)$ and $f_{-}(w)$. In order to evaluate the functions $f_{a}(w), f_{-}(w)$ and $f_{+}(w)$ which determine the solution of (1), we shall need the following easily proved properties:

(i) $f_{a}(w)$ and $g_{a}(w)$ are entire functions of $w$,

(ii) $f_{+}(w)-g_{+}(w)$ is regular for $\operatorname{Im} w>-1$,

(iii) $f_{-}(w)-g_{-}(w)$ is regular for $\operatorname{Im} w<1$,

(iv) $\lambda K(w)-1=\frac{\chi_{-}(w)}{\chi_{+}(w)} \sigma(w)$ for $-1<-\alpha \leq \alpha<1 \quad$ ([1], p. 339 and [2], p. 51) where

(a) $\chi_{-}(w)$ is regular and free of zeros for $\operatorname{Im} w \leq \alpha$,

(b) $m_{-}<\left|\chi_{-}(w) w^{n / 2}\right|<M_{-}$for $\operatorname{Im} w \leq \alpha$, where $m_{-}$and $M_{-}$are positive constants,

(c) $\chi_{+}(w)$ is regular and free of zeros for $\operatorname{Im} w \geq-\alpha$,

(d) $m_{+}<\left|\chi_{+}(w) w^{-n / 2}\right|<M_{+}$for $\operatorname{Im} w \geq-\alpha$, where $m_{+}$and $M_{+}$are positive constants,

(e) $\sigma(w)=\epsilon(w) \epsilon(-w)$ and $\epsilon(w)=\prod_{i=1}^{n / 2}\left(w-w_{i}\right)$, where $n$ is the number of roots $w_{i}$ and $-w_{i}$ of $\lambda K(w)-1$ in $|\operatorname{Im} w| \leq \alpha$.

Using the property (iv), we may rewrite (3) as

$$
\frac{\chi_{-}(w)}{\chi_{+}(w)} \epsilon(w) \epsilon(-w) f_{a}(w)=f_{+}(w)+f_{-}(w)-g_{+}(w)-g_{-}(w)-g_{a}(w) .
$$

The present method depends upon converting (4) into two simultaneous integral equations for $f_{+}(w)$ and $f_{-}(w)$. To obtain the first of these equations, we re-express (4) as

$$
f_{a}(w) \frac{\epsilon(-w)}{\chi_{+}(w)}=\frac{f_{+}(w)-g_{+}(w)-g_{a}(w)}{\epsilon(w) \chi_{-}(w)}+\frac{f_{-}(w)-g_{-}(w)}{\epsilon(w) \chi_{-}(w)},
$$

where from the properties (i), (iii) and (iv) the term on the left-hand side is regular for $\operatorname{Im} w>-\alpha$ and the second term on the right-hand side is regular except at the zeros of $\epsilon(w)$ for $\operatorname{Im} w<\alpha$. We now consider a contour $C_{\alpha}$ which connects the points 
$i \alpha-\infty$ and $i \alpha+\infty$ by a curve which lies in the region $-\alpha<\operatorname{Im} w<\alpha$ and passes below the zeros of $\epsilon(w)$ and a contour $C_{-\alpha}$ which connects the points $-i \alpha-\infty$ and $-i \alpha+\infty$ by a curve lying below $C_{\alpha}$ but above $\operatorname{Im} w=-\alpha$. Then we split the term

$$
\frac{f_{+}(w)-g_{+}(w)-g_{a}(w)}{\epsilon(w) \chi_{-}(w)}
$$

and hence the whole of equation $\left(4^{\prime}\right)$, into two parts, one regular in the half-plane above $C_{-\alpha}$ and the other below $C_{\alpha}$. Thus by Cauchy's theorem

$$
\begin{aligned}
& \frac{f_{+}(w)-g_{+}(w)-g_{a}(w)}{\epsilon(w) \chi_{-}(w)}=\frac{1}{2 \pi i}\left\{\int_{c_{-\alpha}}-\int_{c_{\alpha}}+\int_{-i \alpha+\infty}^{i \alpha+\infty}+\int_{i \alpha-\infty}^{-i \alpha-\infty}\right\} \\
& \frac{d w^{\prime}}{w^{\prime}-w} \frac{f_{+}\left(w^{\prime}\right)-g_{+}\left(w^{\prime}\right)-g_{a}\left(w^{\prime}\right)}{\epsilon\left(w^{\prime}\right) \chi_{-}\left(w^{\prime}\right)}
\end{aligned}
$$

where $w$ lies within the closed contour. From the remark following (3) it follows that the third and fourth integrals on the right-hand side of this equation vanish. Thus

$$
\begin{aligned}
\frac{f_{+}(w)-g_{+}(w)-g_{a}(w)}{\epsilon(w) \chi_{-}(w)} & =\frac{1}{2 \pi i}\left\{\int_{c_{-\alpha}}-\int_{c_{\alpha}}\right\} \frac{d w^{\prime}}{w^{\prime}-w} \frac{f_{+}\left(w^{\prime}\right)-g_{+}\left(w^{\prime}\right)-g_{a}\left(w^{\prime}\right)}{\epsilon\left(w^{\prime}\right) \chi_{-}\left(w^{\prime}\right)} \\
& =I\left(w, C_{-\alpha}\right)-I\left(w, C_{\alpha}\right) .
\end{aligned}
$$

From the definition of $I\left(w, C_{-\alpha}\right)$, we see that this function is regular for $I: \cdots w$ above $C_{-\alpha}$ which results from the uniform convergence of the integral with respect to $w$ in any region whose closure is in the region $\operatorname{Im} w$ above $C_{-\alpha}$. Similarly, $I\left(w, C_{\alpha}\right)$ is regular for $\operatorname{Im} w$ in the half plane below $C_{\alpha}$. Thus (4') may be written

$$
\frac{f_{-}(w)-g_{-}(w)}{\epsilon(w) \chi_{-}(w)}-I\left(w, C_{\alpha}\right)=f_{a}(w) \frac{\epsilon(-w)}{\chi_{+}(w)}-I\left(w, C_{-\alpha}\right) .
$$

From the preceding remarks, the right-hand side of this equation is regular for Im $w$ above $C_{-\alpha}$ and the left-hand side is regular for $\operatorname{Im} w$ below $C_{\alpha}$. Thus (6) defines a function regular in the whole $w$-plane and thus it defines an entire function. From properties (iv) (b) and (d), $1 / \epsilon(w) \chi_{-}(w)$ and $\epsilon(-w) / \chi_{+}(w)$ are bounded for $\operatorname{Im} w$ below $C_{\alpha}$ and above $C_{-\alpha}$, respectively. Further, from Theorems III and V of [2], $f_{-}(w)-g_{-}(w)$ is bounded in every properly included half-plane below $C_{\alpha}$. Analogously, $f_{a}(w)$ is bounded in the half-plane above $C_{-\alpha}$. Finally, from the uniform convergence of $I\left(w, C_{-\alpha}\right)$ and $I\left(w, C_{\alpha}\right)$ with respect to $w$, it follows that these quantities are bounded and approach zero as $|w| \rightarrow \infty$ in the interior of their respective half-planes of regularity. Thus, the two sides of (6) are bounded and, hence, (6) defines a polynomial $p(w)$ of degree at most zero. However, since $f_{-}(w)-g_{-}(w), f_{a}(w), I\left(w, C_{-\alpha}\right)$, and $I\left(w, C_{\alpha}\right)$ vanish as $|\mathrm{Rl} w| \rightarrow \infty$ in their respective regions of regularity, $p(w)$ must be identically zero. Thus

$$
f_{a}(w)=\frac{\chi_{+}(w)}{\epsilon(-w)} \frac{1}{2 \pi i} \int_{c_{-\alpha}} \frac{d w^{\prime}}{w^{\prime}-w} \frac{f_{+}\left(w^{\prime}\right)-g_{+}\left(w^{\prime}\right)-g_{a}\left(w^{\prime}\right)}{\epsilon\left(w^{\prime}\right) \chi_{-}\left(w^{\prime}\right)}
$$

for $\operatorname{Im} w$ above $C_{-\alpha}$ and

$$
\frac{f_{-}(w)-g_{-}(w)}{\epsilon(w) \chi_{-}(w)}=\frac{1}{2 \pi i} \int_{c_{\alpha}} \frac{d w^{\prime}}{w^{\prime}-w} \frac{f_{+}\left(w^{\prime}\right)-g_{+}\left(w^{\prime}\right)-g_{a}\left(w^{\prime}\right)}{\epsilon\left(w^{\prime}\right) \chi_{-}\left(w^{\prime}\right)}
$$

for $\operatorname{Im} w$ below $C_{\alpha}$. 
In analogy with the calculation just described, if we start with (4), rewriting it as

$$
\begin{aligned}
\chi_{-}(w) \epsilon(w) e^{-i a w} f_{a}(w)= & \frac{\chi_{+}(w)}{\epsilon(-w)} e^{-i a w}\left[f_{+}(w)-g_{+}(w)\right] \\
& +\frac{\chi_{+}(w)}{\epsilon(-w)} e^{-i a w}\left[f_{-}(w)-g_{-}(w)-g_{a}(w)\right],
\end{aligned}
$$

then split the second term on the right-hand side into two parts; one regular for Im $w$ below $C_{\beta}$ and the other regular for $\operatorname{Im} w$ above $C_{-\beta}$, where $C_{-\beta}$ connects $-i \beta-\infty$ to $-i \beta+\infty$ by a contour passing above the zeros of $\epsilon(-w)$ and contained in the region $-\beta \operatorname{Im} w<\beta$, and $C_{\beta}$ connects $i \beta-\infty$ to $i \beta+\infty$ and lies above $C_{-\beta}$ in the strip $-\beta<\operatorname{Im} w<\beta$, then we have

$$
f_{a}(w)=-\frac{e^{i a w}}{\chi_{-}(w) \epsilon(w)} \frac{1}{2 \pi i} \int_{c_{\beta}} \frac{d w^{\prime}}{w^{\prime}-w} \frac{f_{-}\left(w^{\prime}\right)-g_{-}\left(w^{\prime}\right)-g_{a}\left(w^{\prime}\right)}{\epsilon\left(-w^{\prime}\right)} \chi_{+}\left(w^{\prime}\right) e^{-i a w^{\prime}}
$$

for $\operatorname{Im} w$ below $C_{B}$ and

$$
\begin{aligned}
{\left[f_{+}(w)-g_{+}(v)\right] \frac{\chi_{+}(w)}{\epsilon(-w)} e^{-i a w}=} & -\frac{1}{2 \pi i} \int_{C-\beta} \\
& \cdot \frac{d w^{\prime}}{w^{\prime}-w} \frac{f_{-}\left(w^{\prime}\right)-g_{-}\left(w^{\prime}\right)-g_{a}\left(w^{\prime}\right)}{\epsilon\left(-w^{\prime}\right)} \chi_{+}\left(w^{\prime}\right) e^{-i a w^{\prime}}
\end{aligned}
$$

for $\operatorname{Im} w$ above $C_{-\beta}$. The presence of $e^{-i a w}$ and the specific arrangement of $g_{\odot}(W)$ in $\left(4^{\prime \prime}\right)$ are required in order to bound the two sides of $\left(4^{\prime \prime}\right)$ according to the argument presented after (6); in particular, for this purpose, it is necessary to have the easily derived results that $\left|e^{-i a w} f_{a}(w)\right|=o(1)$ as $\operatorname{Im} w \rightarrow-\infty$ and $\left|e^{-i a w}\left[f_{+}(w)-g_{+}(w)\right]\right|=$ $o(1)$ as $\operatorname{Im} w \rightarrow \infty$.

The important result is the pair of integral equations (8) and (10). The solutions of these equations, when combined with (7), determine $f_{a}(w)$ and finally $f(x)$. Unfortunately, exact solutions can only be obtained for the case that $K(w)$ is a rational fraction in $w$. However, approximate solutions can be readily obtained.

4. Approximate solutions of (8) and (10). To solve (8) and (10) approximately we let the contour $C_{\alpha}$ be shifted toward $+i \infty$ and the contour $C_{-\beta}$ be shifted toward $-i \infty$. The contours sweep across the singularities of the integrands of (8) and (10). The singularities are easily seen to be poles, except possibly for essential singularities arising from $\chi_{+}(w)$ and $\chi_{-}(w)$. If such essential singularities exist, they may be removed from the finite part of the plane by a simple device. Namely, since only values of $k(x)$ in the interval $-a \leq x \leq a$ enter into (1), $k(x)$ may be chosen zero outside this interval as remarked in Sec. 2. As a consequence, $K(w)$ becomes an entire function and, therefore, $\chi_{+}(w)$ and $\chi_{-}(w)$ have no essential singularities in the finite part of the $w$-plane. Thus if the contours are moved to infinity, the only singularities crossed will be poles. In this manner, (8) and (10) may be written

$$
\begin{gathered}
\frac{f_{-}(w)-g_{-}(w)}{\epsilon(w) \chi_{-}(w)}=r(w)+\sum \operatorname{Res}\left(\frac{f_{+}\left(w^{\prime}\right)}{\epsilon\left(w^{\prime}\right) \chi_{-}\left(w^{\prime}\right)} \frac{1}{w^{\prime}-w}\right)+R\left(w, \alpha^{\prime}\right), \\
{\left[f_{+}(w)-g_{+}(w)\right] \frac{\chi_{+}(w)}{\epsilon(-w)} e^{-i a w}=s(w)+\sum \operatorname{Res} \cdot\left(\frac{f_{-}\left(w^{\prime}\right) \chi_{+}\left(w^{\prime}\right)}{\epsilon\left(-w^{\prime}\right)} \frac{e^{-i a w^{\prime}}}{w^{\prime}-w}\right)+S\left(w, \beta^{\prime}\right),}
\end{gathered}
$$


where

$$
\begin{aligned}
& r(w)=-\frac{1}{2 \pi i} \int_{c_{\alpha}} \frac{d w^{\prime}}{w^{\prime}-w} \frac{g_{+}\left(w^{\prime}\right)+g_{a}\left(w^{\prime}\right)}{\epsilon\left(w^{\prime}\right) \chi_{-}\left(w^{\prime}\right)} \\
& s(w)=\frac{1}{2 \pi i} \int_{c_{-\beta}} \frac{d w^{\prime}}{w^{\prime}-w} \frac{\left[g_{-}\left(w^{\prime}\right)+g_{a}\left(w^{\prime}\right)\right] \chi_{+}\left(w^{\prime}\right)}{\epsilon\left(-w^{\prime}\right)} e^{-i a w^{\prime}} \\
& R\left(w, \alpha^{\prime}\right)=\frac{1}{2 \pi i} \int_{i \alpha^{\prime}-\infty}^{i \alpha^{\prime}+\infty} \frac{d w^{\prime}}{w^{\prime}-w} \frac{f_{+}\left(w^{\prime}\right)}{\epsilon\left(w^{\prime}\right) \chi_{-}\left(w^{\prime}\right)} \\
& S\left(w, \beta^{\prime}\right)=-\frac{1}{2 \pi i} \int_{-i \beta^{\prime}-\infty}^{-i \beta^{\prime}+\infty} \frac{d w^{\prime}}{w^{\prime}-w} \frac{f_{-}\left(w^{\prime}\right) \chi_{+}\left(w^{\prime}\right)}{\epsilon\left(-w^{\prime}\right)} e^{-i a w^{\prime}}
\end{aligned}
$$

In (11) and (12) the summations are over the residues at the poles of the integrands crossed by the contours as $C_{\alpha}$ is moved up to $i \alpha^{\prime}$ and $C_{-\beta}$ is moved down to $-i \beta^{\prime}$. Approximate solutions of (8) and (10) are therefore provided by (11) and (12) if $R\left(w, \alpha^{\prime}\right)$ and $S\left(w, \beta^{\prime}\right)$ are neglected. The residues in (11) and (12) are obtained in this approximation in terms of the approximation itself by solving an obvious system of linear equations derived from (11) and (12). In the case that the contours $C_{\alpha}$ and $C_{-\beta}$ sweep across multiple poles, the residues of (11) and (12) involve derivatives of $f_{+}(w)$ and $f_{-}(w)$. These derivatives may be evaluated by differentiating (11) and (12), and including the resultant equations into an extended system of linear equations for determining the residues.

A question may be raised with regard to the uniqueness and convergence of the residues obtained from the solution of the linear system derived from (11) and (12). It has not been possible as yet to establish rigorously and generally this uniqueness and convergence. In a number of simple applications of the present method, in particular to cases wherein $K(w)$ is a rational fraction, the linear system has been found to have unique solutions; that is, the determinant of the system was non-singular except at eigenvalues. Moreover, for $K(w)$ a rational fraction, convergence was automatic, since the determinant was of finite order and an exact solution was obtainable. However, since the determinant of the linear system involves only $\chi_{+}(w), \chi_{-}(w)$ and $\epsilon(w)$, and hence depends only upon $K(w)$, and, moreover, is non-singular except at eigenvalues for some forms of $K(w)$, it is a reasonable presumption that the determinant has no special degeneracy and that only exceptional forms for $K(w)$ will lead to non-uniqueness or non-convergence.

Finally an estimate of the accuracy of the approximation for $f_{+}(w)$ and $f_{-}(w)$ may be obtained by estimating the order of magnitude of the terms $R\left(w, \alpha^{\prime}\right)$ and $S\left(w, \beta^{\prime}\right)$. Using the easily proved results that $\left|f_{+}(w) e^{-i a w}\right| \rightarrow 0$ as $\operatorname{Im} w \rightarrow \infty$ and $\left|f_{-}(w)\right| \rightarrow 0$ as $\operatorname{Im} w \rightarrow-\infty$, we find that

$$
\left|R\left(w, \alpha^{\prime}\right)\right|<K e^{-a \alpha^{\prime}}
$$

and

$$
\left|S\left(w, \beta^{\prime}\right)\right|<M e^{-a \beta^{\prime}}
$$

where $K$ and $M$ are constants. Thus the accuracy of the approximation improves exponentially with increasing $\alpha^{\prime}$ and $\beta^{\prime}$.

The corresponding approximation for $f_{0}(w)$ is determined by substituting (8) and (10) into (4), after neglecting $R\left(w, \alpha^{\prime}\right)$ and $S\left(w, \beta^{\prime}\right)$. 
5. Approximation for $f(x)$. From the Fourier inversion formula and from (4), we we find that, for $0 \leq x \leq a, f(x)$ is given ly

$$
\begin{aligned}
& f(x)=\frac{1}{2 \pi} \int_{-i \beta-\infty}^{-i \beta+\infty} d w^{\prime} e^{-i x w^{\prime}} f_{a}\left(w^{\prime}\right) \\
& =\frac{1}{2 \pi} \int_{-i \beta-\infty}^{-i \beta+\infty} d w^{\prime} e^{-i x w^{\prime}}\left[\frac{e^{i a w^{\prime}}}{\epsilon\left(w^{\prime}\right) \chi_{-}}\left(w^{\prime}\right)\left(\frac{f_{+}\left(w^{\prime}\right) \dot{\chi}_{+}\left(w^{\prime}\right) e^{-i a w^{\prime}}}{\epsilon\left(-w^{\prime}\right)}\right)\right. \\
& \left.+\frac{\chi_{+}\left(w^{\prime}\right)}{\epsilon\left(-w^{\prime}\right)}\left(\frac{f_{-}\left(w^{\prime}\right)}{\epsilon\left(w^{\prime}\right) \chi_{-}\left(w^{\prime}\right)}\right)-\frac{\chi_{1}}{\chi} \frac{\left(w^{\prime}\right)}{\left(w^{\prime}\right)} \frac{g_{+}\left(w^{\prime}\right)+g_{-}\left(w^{\prime}\right)+g_{a}\left(w^{\prime}\right)}{\epsilon\left(w^{\prime}\right) \epsilon\left(-w^{\prime}\right)}\right],
\end{aligned}
$$

where $-\infty<\beta<\infty$. For our purposes, we restrict $\beta$ so that the contour in this equation lies below the zeros of $\epsilon(w)$ and $\chi_{-}(w)$, below the essential singularities, if any, of $\chi_{-}(w)$ and below the singularities of $g_{-}(w)$, but above the zeros of $\epsilon(-w)$ and poles of $\chi_{+}(w)$, above the singularities of $g_{+}(w)$, and above any essential singularities of $\chi_{+}(w)$. [For convenience it is assumed that $w=0$ is not a root of $\epsilon(w)]$. Now in the first integral on the right-hand side of (13) we allow $-\beta$ to increase to, say, $\alpha^{\prime}$; in the second integral we allow $-\beta$ to decrease to, say, $-\beta^{\prime}$. This procedure leads to the following expression for $f(x)$ :

$$
\begin{aligned}
& f(x)=i \sum \operatorname{Res} .\left[\frac{e^{i(a-x) w^{\prime}}}{\epsilon\left(w^{\prime}\right) \chi_{-}\left(w^{\prime}\right)}\left(\frac{j_{1}\left(w^{\prime}\right)}{\epsilon\left(-w^{\prime}\right)}\right)\right]+I\left(x, \alpha^{\prime}\right) \\
& -i \sum \operatorname{Res} .\left[\frac{e^{-i x w^{\prime}} \chi_{+}\left(w^{\prime}\right)}{\epsilon\left(-w^{\prime}\right)}\left(\frac{f}{\epsilon\left(w^{\prime}\right)} \frac{-\left(w^{\prime}\right)}{\chi_{-}\left(w^{\prime}\right)}\right)\right]+J\left(x, \beta^{\prime}\right) \\
& -\frac{1}{2 \pi} \int_{-i \beta-\infty}^{-i \beta+\infty} d w^{\prime} e^{-i x w^{\prime}} \frac{g_{+}\left(w^{\prime}\right)+g}{\epsilon\left(w^{\prime}\right) \epsilon\left(-w^{\prime}\right) \chi_{-}\left(w^{\prime}\right)} \chi_{+}\left(w^{\prime}\right),
\end{aligned}
$$

where

$$
\begin{aligned}
& I\left(x, \alpha^{\prime}\right)=\frac{1}{2 \pi} \int_{i \alpha^{\prime}-\infty}^{i \alpha^{\prime}+\infty} d w^{\prime} \frac{e^{i(a-x) w^{\prime}}}{\epsilon\left(w^{\prime}\right) \chi \cdot\left(w^{\prime}\right)}\left(\frac{f_{+}\left(w^{\prime}\right) \chi_{+}\left(w^{\prime}\right)}{\epsilon\left(-w^{\prime}\right)} e^{-i a w^{\prime}}\right), \\
& J\left(x, \beta^{\prime}\right)=\frac{1}{2 \pi} \int_{-i \beta^{\prime}-\infty}^{-i \beta^{\prime}+\infty} d w^{\prime} e^{-i x w^{\prime}} \frac{\chi_{1}\left(w^{\prime}\right)}{\epsilon\left(-w^{\prime}\right)}\left(\frac{f_{-}\left(w^{\prime}\right)}{\epsilon\left(w^{\prime}\right) \chi_{-}\left(w^{\prime}\right)}\right),
\end{aligned}
$$

and $0 \leq x \leq a$. The summations in (14) are over the residues at the poles crossed by the contours as $-\beta$ is increased to $\alpha^{\prime}$ in the first integral of (13) and is decreased to $-\beta^{\prime}$ in the second integral.

The final approximation to $f(x)$ is obtained by neglecting $I\left(x, \alpha^{\prime}\right)$ and $J\left(x, \beta^{\prime}\right)$ in (14) and by using the expressions (11) and (12), neglecting $R\left(w, \alpha^{\prime}\right)$ and $S\left(w, \beta^{\prime}\right)$, for $f_{+}(w)$ and $f_{-}(w)$. A straightforward calculation shows that the error resulting from neglect of $I\left(x, \alpha^{\prime}\right)$ and $J\left(x, \beta^{\prime}\right)$ is

$$
\left|I\left(x, \alpha^{\prime}\right)\right|<A e^{-(a-x) \alpha^{\prime}}
$$

and

$$
\left|J\left(x, \beta^{\prime}\right)\right|<B e^{-\beta^{\prime} x},
$$

where $A$ and $B$ are constants. Thus for large $\alpha^{\prime}$ and $\beta^{\prime}$, the error is small except in the neighborhood of $x=0$ and $x=a$, which for increasing $a$ becomes less and less important. 
6. Eigenvalues of (1). In the case that $g(x)=0$ in (1), we have an eigenvalue problem. Then (11) and (12) become a system of linear homogeneous equations for the determination of the values of $f_{+}(w)$ and $f_{-}(w)$ at the poles of the integrands of (8) and (10). The values of $\lambda$ admitting of solutions of (11) and (12) under these circumstances will clearly then constitute approximate eigenvalues for (1).

\section{Example:}

$$
f(x)=e^{-\alpha|x|}+\lambda \int_{0}^{a} d y e^{-|x-y|} f(y) .
$$

To illustrate the application of the present method, the above simple integral equation will be treated. This equation admits of an exact solution since the transform of the kernel is a rational fraction and, consequently, the series expansions in (11), (12) and (14) terminate.

From the definitions (i)-(vii) of Sec. 2 and property (iv) of Sec. 3, we find:

$$
\begin{aligned}
g_{-}(w) & =\frac{1}{\alpha+i w}, \\
g_{+}(w) & =\frac{e^{a(i w-\alpha)}}{\alpha-i w}, \\
g_{a}(w) & =\frac{1-e^{a(i w-\alpha)}}{\alpha-i w}, \\
\lambda K(w)-1 & =\frac{2 \lambda-1-w^{2}}{w^{2}+1}, \\
\epsilon(w) & =w-(2 \lambda-1)^{1 / 2}, \\
\chi_{+}(w) & =w+i, \\
\chi_{-}(w) & =\frac{1}{w-i} .
\end{aligned}
$$

Substitution of these quantities into (11) and (12) and evaluation of the residues leads to the result

$$
\begin{aligned}
f_{-}(w)=\frac{1}{\alpha+i w}+ & \left\{\frac{(2 \lambda-1)^{1 / 2}-i}{\alpha-i(2 \lambda-1)^{1 / 2}}+\left[(2 \lambda-1)^{1 / 2}-i\right] f_{+}\left([2 \lambda-1]^{1 / 2}\right)\right\} \frac{1}{w-i}, \\
f_{+}(w)=\frac{e^{a(i w-\alpha)}}{\alpha-i w}+ & \left\{\left[-(2 \lambda-1)^{1 / 2}+i\right]\right. \\
& \cdot\left(\frac{1}{\alpha-i(2 \lambda-1)^{1 / 2}}+\frac{1-\exp \left\{-a\left[\alpha+i(2 \lambda-1)^{1 / 2}\right]\right\}}{\alpha+i(2 \lambda-1)^{1 / 2}}\right) \\
& \cdot \exp \left[i a(2 \lambda-1)^{1 / 2}\right]+\left[(2 \lambda-1)^{1 / 2}-i\right] f_{-}\left(-[2 \lambda-1]^{1 / 2}\right) \\
& \left.\cdot \exp \left[i a(2 \lambda-1)^{1 / 2}\right]\right\} \frac{e^{i a \omega}}{w+i}
\end{aligned}
$$


Forming from these equations the linear system for evaluating $f_{+}\left([2 \lambda-1]^{1 / 2}\right)$ and $f_{-}\left(-[2 \lambda-1]^{1 / 2}\right)$ and carrying out the calculation gives

$$
\begin{aligned}
f_{+}\left([2 \lambda-1]^{1 / 2}\right)= & \frac{1}{D} \frac{i(2 \lambda-1)^{1 / 2}}{\lambda\left(\alpha^{2}+2 \lambda-1\right)}\left\{\frac{1+\alpha}{i(2 \lambda-1)^{1 / 2}-1} \exp \left[i a(2 \lambda-1)^{1 / 2}\right]\right. \\
& \left.\quad+\frac{1-\alpha}{i(2 \lambda-1)^{1 / 2}+1} e^{-a \alpha}\right\}, \\
f_{-}\left(-[2 \lambda-1]^{1 / 2}\right)= & \frac{1}{D} \frac{1}{\lambda\left(\alpha^{2}+2 \lambda-1\right)}\left\{\alpha \frac{i(2 \lambda-1)^{1 / 2}+1}{i(2 \lambda-1)^{1 / 2}-1} \exp \left[i a(2 \lambda-1)^{1 / 2}\right]\right. \\
+ & \left.\frac{\alpha+i(2 \lambda-1)^{1 / 2}}{i(2 \lambda-1)^{1 / 2}+1} \exp \left[-i a(2 \lambda-1)^{1 / 2}\right]+\frac{i(2 \lambda-1)^{1 / 2}(1-\alpha)}{i(2 \lambda-1)^{1 / 2}-1} e^{-a \alpha}\right\},
\end{aligned}
$$

where the determinant, $D$, of the linear system is given by

$$
D=\frac{\exp \left[-i a(2 \lambda-1)^{1 / 2}\right]}{\left(i[2 \lambda-1]^{1 / 2}+1\right)^{2}}-\frac{\exp \left[i a(2 \lambda-1)^{1 / 2}\right]}{\left(i[2 \lambda-1]^{1 / 2}-1\right)^{2}}
$$

The values of $\lambda$ for which $D=0$ are the eigenvalues. For the present case, $\lambda=1 / 2$ is the smallest eigenvalue.

For $0 \leq x \leq a$, evaluation of the residues in (14) yields

$$
\begin{aligned}
f(x)= & -\frac{i \lambda}{(2 \lambda-1)^{1 / 2}} f_{+}\left([2 \lambda-1]^{1 / 2}\right) \exp \left[-i x(2 \lambda-1)^{1 / 2}\right] \\
& -\frac{i \lambda}{(2 \lambda-1)^{1 / 2}}\left\{f_{-}\left(-[2 \lambda-1]^{1 / 2}\right)-\frac{2 \alpha}{\alpha^{2}+2 \lambda-1}\right\} \exp \left[i x(2 \lambda-1)^{1 / 2}\right] \\
& +\frac{\alpha^{2}-1}{\alpha^{2}+2 \lambda-1} e^{-\alpha x} .
\end{aligned}
$$

If $D \neq 0$, the above expressions for $f_{+}\left([2 \lambda-1]^{1 / 2}\right)$ and $f_{-}\left(-[2 \lambda-1]^{1 / 2}\right)$ may be substituted into this equation for $f(x)$, which after rearranging gives the final result

$$
\begin{aligned}
f(x)=\left\{\frac{1+\alpha}{i(2 \lambda-1)^{1 / 2}-1} \exp \left[i a(2 \lambda-1)^{1 / 2}\right]\right. & \left.+\frac{1-\alpha}{i(2 \lambda-1)^{1 / 2}+1} e^{-\alpha \alpha}\right\} \\
& \frac{\exp \left[-i x(2 \lambda-1)^{1 / 2}\right]}{D\left(\alpha^{2}+2 \lambda-1\right)}+\left\{\frac{1+\alpha}{i(2 \lambda-1)^{1 / 2}+1} \exp \left[-i a(2 \lambda-1)^{1 / 2}\right]\right. \\
+ & \left.\frac{1-\alpha}{i(2 \lambda-1)^{1 / 2}-1} e^{-\alpha \alpha}\right\} \frac{\exp \left[i x(2 \lambda-1)^{1 / 2}\right]}{D\left(\alpha^{2}+2 \lambda-1\right)}+\frac{\alpha^{2}-1}{\alpha^{2}+2 \lambda-1} e^{-\alpha z} .
\end{aligned}
$$

This simple example is typical of the application of the present method to equations for which $K(w)$ is a rational fraction. For other functions $K(w)$, the procedure is completely analogous, but has the same difficulty as with the usual Wiener-Hopf method that the functions $\chi_{+}(w)$ and $\chi_{-}(w)$ are in general not evaluable analytically but must be treated either numerically or approximately.

8. Example:

$$
f(x)=\frac{\lambda}{(2 \pi)^{1 / 2}} \int_{0}^{a} d y \exp \left[-\frac{1}{2}(x-y)^{2}\right] f(y)
$$


For the case of the Gaussian kernel, the present method has been applied to the determination of the lowest eigenvalue. Previous consideration [3] of this problem has led to the calculation of upper and lower bounds for this eigenvalue. The present calculation, while illustrating the above method and indicating its accuracy and limitations, provides an extension of the earlier work on this problem by obtaining an accurate estimate of the eigenvalue in the region where the bounding values differ significantly.

Taking the transform of $k(x)=1 /(2 \pi)^{1 / 2} \exp \left(-x^{2} / 2\right)$, we find

$$
\lambda K(w)-1=\lambda \exp \left(-\frac{1}{2} w^{2}\right)-1 .
$$

The roots of this expression occur at

$$
w_{n}= \pm[2 \ln \lambda+4 \pi n i]^{1 / 2},
$$

where $n$ is a positive or negative integer or zero and it is known [3] that $\lambda \geq 1$. For the approximation described below, we limit $\lambda$ so that only the two roots with $n=0$ lie within the strip $|\operatorname{Im} w|<1$. This requires that $\lambda \lesssim 20$. With this restriction, we may write

$$
[\lambda K(w)-1]=\frac{\chi_{-}(w)}{\chi_{+}(w)}\left[-w-(2 \ln \lambda)^{1 / 2}\right]\left[w-(2 \ln \lambda)^{1 / 2}\right] .
$$

From ([1], p. 339) it follows that

and

$$
\chi_{-}(w)=\frac{\exp \left[-\tau_{-}(w)\right]}{w-i}
$$

where

$$
\chi_{+}(w)=-\frac{1}{\chi_{-}(-w)}
$$

$$
\tau_{-}(w)=\frac{1}{2 \pi i} \int_{i \alpha-\infty}^{i \alpha+\infty} \frac{d w^{\prime}}{w^{\prime}-w} \ln \left\{\left[1-\lambda \exp \left(-\frac{1}{2}{w^{\prime 2}}^{2}\right)\right] \frac{{w^{\prime}}^{2}+1}{w^{\prime 2}-2 \ln \lambda}\right\}
$$

and the contour lies in the strip $0<\operatorname{Im} w<1$.

From (11) and (12), it is found that

$$
\begin{gathered}
\frac{f_{-}(w)}{\epsilon(w) \chi_{-}(w)}=\frac{f_{+}\left([2 \ln \lambda]^{1 / 2}\right)}{\chi_{-}\left([2 \ln \lambda]^{1 / 2}\right.} \frac{1}{(2 \ln \lambda)^{1 / 2}-w}, \\
f_{+}(w) \frac{\chi_{+}(w)}{\epsilon_{-}(w)} e^{-i a w}=\frac{f_{-}\left(-[2 \ln \lambda]^{1 / 2}\right) \chi_{+}\left(-[2 \ln \lambda]^{1 / 2}\right) \exp \left[i a(2 \ln \lambda)^{1 / 2}\right]}{(2 \ln \lambda)^{1 / 2}+w},
\end{gathered}
$$

where residues at the roots $w_{n}, n \neq 0$, of $\lambda K(w)-1$ have been neglected. These terms, as argued at the end of Sec. 4 , are of the order of $\exp \left[-a \mid \operatorname{Im}(2 \ln \lambda \pm 4 \pi i)^{1 / 2}\right]$. For $a \sim 1$, it may be shown from [3] that $\lambda \sim 2.5$ so that $\left|\operatorname{Im}(2 \ln \lambda \pm 4 \pi i)^{1 / 2}\right| \sim 2.3$. The error is then of order $e^{-2.3} \sim 1 / 10$ and decreases rapidly as $a$ increases.

The linear system for determining $f_{+}\left([2 \ln \lambda]^{1 / 2}\right)$ and $f_{-}\left(-[2 \ln \lambda]^{1 / 2}\right)$ may now be written

$$
\frac{f_{-}\left(-[2 \ln \lambda]^{1 / 2}\right)}{\chi_{-}\left(-[2 \ln \lambda]^{1 / 2}\right)}-\frac{f_{+}\left([2 \ln \lambda]^{1 / 2}\right)}{\chi_{-}\left([2 \ln \lambda]^{1 / 2}\right)}=0,
$$

$f_{-}\left(-[2 \ln \lambda]^{1 / 2}\right) \chi_{+}\left(-[2 \ln \lambda]^{1 / 2}\right) \exp \left[i a(2 \ln \lambda)^{1 / 2}\right]$

$$
+f_{+}\left([2 \ln \lambda]^{1 / 2}\right) \chi_{+}\left([2 \ln \lambda]^{1 / 2}\right) \exp \left[-i a(2 \ln \lambda)^{1 / 2}\right]=0 .
$$


The eigenvalues are determined by the solubility of this linear system which leads to the vanishing of the determinant of the system, namely,

$$
\frac{\chi_{+}\left([2 \ln \lambda]^{1 / 2}\right)}{\chi_{-}\left(-[2 \ln \lambda]^{1 / 2}\right)} \exp \left[-i a(2 \ln \lambda)^{1 / 2}\right]-\frac{\chi_{+}\left(-[2 \ln \lambda]^{1 / 2}\right)}{\chi_{-}\left([2 \ln \lambda]^{1 / 2}\right)} \exp \left[i a(2 \ln \lambda)^{1 / 2}\right]=0 .
$$

From the definitions of $\chi_{+}(w)$ and $\chi_{-}(w)$, we may re-express this equation as

$$
\exp \left[i 2 a(2 \ln \lambda)^{1 / 2}\right]=\left(\frac{(2 \ln \lambda)^{1 / 2}+i}{(2 \ln \lambda)^{1 / 2}-i}\right)^{2} \exp \left[-2\left\{\tau_{-}\left([2 \ln \lambda]^{1 / 2}\right)-\tau_{-}\left(-[2 \ln \lambda]^{1 / 2}\right)\right\}\right] .
$$

As a further simplification the expression for $\tau_{-}\left([2 \ln \lambda]^{1 / 2}\right)$ may be written

$$
\tau_{-}\left([2 \ln \lambda]^{1 / 2}\right)=-\frac{1}{2} \ln \frac{2 \ln \lambda+1}{2}+\frac{1}{2 \pi i} J\left([2 \ln \lambda]^{1 / 2}\right),
$$

where

$$
J\left([2 \ln \lambda]^{1 / 2}\right)=P \int_{-\infty}^{\infty} \ln \left\{\left[1-\lambda \exp \left(-\frac{1}{2} x^{2}\right)\right] \frac{x^{2}+1}{x^{2}-2 \ln \lambda}\right\} \frac{d x}{x-(2 \ln \lambda)^{1 / 2}}
$$

and the integration is along the real axis, $P$ standing for "principal value." Then the above expression becomes

$$
\exp \left[i a(2 \ln \lambda)^{1 / 2}\right]=\left(\frac{(2 \ln \lambda)^{1 / 2}+i}{(2 \ln \lambda)^{1 / 2}-i}\right)^{2} \exp \frac{-4 J\left([2 \ln \lambda]^{1 / 2}\right)}{2 \pi i} .
$$

A simple argument shows that this yields

$$
a=\frac{2 \tan ^{-1} \frac{1}{(2 \ln \lambda)^{1 / 2}}+\frac{1}{\pi} J\left([2 \ln \lambda]^{1 / 2}\right)+n \pi}{(2 \ln \lambda)^{1 / 2}}
$$

where $n$ is a positive or negative integer or zero. It may be shown that as $\lambda \rightarrow 1$, $J\left([2 \ln \lambda]^{1 / 2}\right) \rightarrow 0$ as $2 \ln \lambda$. Thus, since for the lowest eigenvalue $a \rightarrow \infty$ as $\lambda \rightarrow 1$, it is easily seen that $n=0$. The larger eigenvalues correspond to $n>0$.

An evaluation of $J\left([2 \ln \lambda]^{1 / 2}\right)$ was carried out numerically so as to exhibit the values of $\lambda$ as a function of $a$. The results are contained in the following table.

\begin{tabular}{cccc}
$a$ & $\lambda$ & $I_{L}$ & $I_{U}$ \\
\hline 6.03 & 1.10 & 1.00 & 1.15 \\
4.03 & 1.20 & 1.05 & 1.25 \\
2.65 & 1.40 & 1.23 & 1.43 \\
2.05 & 1.60 & 1.44 & 1.62 \\
1.70 & 1.80 & 1.65 & 1.81 \\
1.46 & 2.00 & 1.87 & 2.01 \\
1.10 & 2.50 & 2.39 & 2.50 \\
.891 & 3.00 & 2.91 & 3.00 \\
.747 & 3.50 & 3.43 & 3.51 \\
.641 & 4.00 & 3.98 & 4.04 \\
.559 & 4.50 & 4.54 & 4.60 \\
.492 & 5.00 & 5.14 & 5.19 \\
.390 & 6.00 & 6.47 & 6.51 \\
.253 & 8.00 & 9.93 & 9.93
\end{tabular}


The quantities $I_{L}$ and $I_{U}$ are

$$
\begin{aligned}
& I_{L}=\left\{\frac{2}{(2 \pi)^{1 / 2}} \int_{0}^{a / 2} \exp \left(-\frac{1}{2} x^{2}\right) d x\right\}^{-1}, \\
& I_{U}=\left\{\frac{2}{(2 \pi)^{1 / 2}} \int_{0}^{a} \exp \left(-\frac{1}{2} x^{2}\right) d x-\frac{2}{a(2 \pi)^{1 / 2}}\left[1-\exp \left(-\frac{1}{2} a^{2}\right)\right]\right\}^{-1},
\end{aligned}
$$

and correspond to the lower and upper bounds of the eigenvalue deduced in [3]. It is observed that to three figures the eigenvalue obtained above lies within the bounds $I_{L}$ and $I_{U}$ for $a \gtrsim 0.6$ which is consistent with the earlier error estimate. For smaller values of $a$ the present approximation begins to break down as expected.

\section{REFERENCES}

1. E. C. Titchmarsh, Fourier integrals, Oxford Press, 1937

2. R. Paley and N. Wiener, Fourier transforms in the complex domain, Amer. Math. Soc. Coll. Publ., 1934

3. R. Bellman and R. Latter, Proc. Amer. Math. Soc. 3, 884 (1952) 